\title{
Editorial: Mechanical Metamaterials: Cutting-Edge Metastructures
}

\author{
Jaehyung $\mathrm{Ju}^{1 *}$ and Xin-Lin $\mathrm{Gao}^{2 *}$ \\ ${ }^{1}$ UM-SJTU Joint Institute, Shanghai Jiao Tong University, Shanghai, China, ${ }^{2}$ Department of Mechanical Engineering, Southern \\ Methodist University, Dallas, TX, United States
}

Keywords: mechanical metamaterials, metastructures, vibrations attenuation, machine learning, morphing wings

\section{Editorial on the Research Topic}

\section{Mechanical Metamaterials: Cutting-Edge Metastructures}

OPEN ACCESS

Edited and reviewed by: Guoqiang Li,

Louisiana State University, United States

*Correspondence: Jaehyung Ju jaehyung.ju@sjtu.edu.cn

Xin-Lin Gao xlgao@smu.edu

Specialty section: This article was submitted to Solid and Structural Mechanics,

a section of the journal

Frontiers in Mechanical Engineering

Received: 02 February 2022 Accepted: 08 February 2022

Published: 25 February 2022

Citation:

Ju J and Gao X-L (2022) Editorial: Mechanical Metamaterials: Cutting-

Edge Metastructures.

Front. Mech. Eng 8:868418. doi: 10.3389/fmech.2022.868418
While organizing this special topic, we invited papers on mechanical metamaterials that are artificially designed material systems with exotic properties rarely found in nature. The exciting features of mechanical metamaterials are usually defined by their topological architecture and microstructural connectivity rather than composition. We received three papers on mechanical metamaterials - two for fundamental research (static and dynamic) and one for applied research: 1) Anigbogu et al., Layered Metamaterial Beam Structure With Local Resonators for Vibration Attenuation: Model and Experiment., 2) Challapalli et al., Discovery of Cellular Unit Cells With High Natural Frequency and Energy Absorption Capabilities by an Inverse Machine Learning Framework, and 3) Xiao et al., A New Architecture of Morphing Wing Based on Hyperelastic Materials and Metastructures With Tunable Stiffness.

Anigbogu et al. investigated vibration attenuation of layered metamaterials with local resonators, demonstrating an increase and shift of the low-frequency bandgap with local resonators. Their work opens a new strategy for vibration mitigation by using local resonators.

Challapalli et al. utilized machine learning to find cellular (lattice) structures with a high natural frequency to avoid resonance and high impact energy, demonstrating a 30\%-100\% higher natural frequency and a 300\% improvement in energy absorption. Their work shows the potential impact of machining learning on the design of metamaterials.

Xiao et al. studied a new metastructure for morphing wing design to break through the technical challenge of small-scale aircraft. Their morphing wing design with soft pneumatic actuators and modified pantographic metastructures demonstrates spanwise and chordwise morphing capabilities, showing an engineering breakthrough of prototyping.

These papers highlight some current engineering challenges of metamaterial design. This community will grow fast and impact the world through scientific findings and 
engineering breakthroughs. We hope that readers will enjoy reading these papers specially solicited for the community.

\section{AUTHOR CONTRIBUTIONS}

JJ drafted the initial manuscript. X-LG edited the manuscript.

Conflict of Interest: The authors declare that the research was conducted in the absence of any commercial or financial relationships that could be construed as a potential conflict of interest.
Publisher's Note: All claims expressed in this article are solely those of the authors and do not necessarily represent those of their affiliated organizations, or those of the publisher, the editors and the reviewers. Any product that may be evaluated in this article, or claim that may be made by its manufacturer, is not guaranteed or endorsed by the publisher.

Copyright (c) $2022 \mathrm{Ju}$ and Gao. This is an open-access article distributed under the terms of the Creative Commons Attribution License (CC BY). The use, distribution or reproduction in other forums is permitted, provided the original author $(s)$ and the copyright owner(s) are credited and that the original publication in this journal is cited, in accordance with accepted academic practice. No use, distribution or reproduction is permitted which does not comply with these terms. 